\title{
Sphingomyelin synthase is absent from endosomes
}

\author{
Ardy van Helvoort ${ }^{1, \star}$, Willem Stoorvogel ${ }^{1}$, Gerrit van Meer ${ }^{1,2}$ and Koert N. J. Burger ${ }^{1}$ \\ ${ }^{1}$ Department of Cell Biology, Faculty of Medicine and Institute of Biomembranes, Universiteit Utrecht, Utrecht, The Netherlands \\ 2Laboratory of Cell Biology and Histologie, University of Amsterdam, Academic Medical Center, Amsterdam, The Netherlands
}

*Author for correspondence at present address: Department of Molecular Biology, The Netherlands Cancer Institute, Plesmanlaan 121, 1066 CX, Amsterdam, The Netherlands (e-mail: helvoort@nki.nl)

\section{SUMMARY}

Both the Golgi and the endosomes have recently been proposed as the main site of SM-synthase, the enzyme responsible for sphingomyelin (SM) biosynthesis. To settle this confusion, we studied the subcellular distribution of SM-synthase in human liver-derived HepG2 and baby hamster kidney BHK-21 cells. To discriminate between Golgi and endosomes we made use of 3,3-diaminobenzidine (DAB) cytochemistry. Cells were incubated with a conjugate of transferrin (Tf) and horseradish peroxidase (HRP), or with unconjugated HRP, to label the recycling pathway and the complete endocytic pathway (including lysosomes) with peroxidase activity, respectively. After cell homogenization, the peroxidase activity was used to induce a local deposition of DAB-polymer. The total SM-synthase activity was not affected by this procedure, and, in contrast to endosomes labeled with ${ }^{125}$ I-Tf, organelles containing SM-synthase did not increase in buoyant density as determined by Percoll density gradient fractionation. Thus, little, if any, SM-synthase localizes to the endocytic pathway of HepG2 and BHK-21 cells. In experiments performed at low temperature to inhibit vesicular transport, we found less than $10 \%$ of newly synthesized short-chain SM at the cell surface. We conclude that most SM-synthase activity is present in the Golgi, and to a small extent at the cell surface.

Key words: Sphingolipid, DAB-cytochemistry, Endosome, Plasma membrane

\section{INTRODUCTION}

Sphingomyelin (SM) is a membrane lipid unique to animal cells. It is virtually restricted to the lumenal leaflet of the organelles of the secretory and endocytic pathways. SM is not equally distributed among these organelles, most SM resides in the exoplasmic leaflet of the plasma membrane, while the ER, for example, contains hardly any SM. It has been proposed that SM in the cytoplasmic leaflet of the plasma membrane is involved in signal transduction and is hydrolyzed upon cell activation (Linardic and Hannun, 1994; Andrieu et al., 1996). To understand the differential distribution of SM within the cell, and the enrichment of SM at the cell surface, it is essential to identify the site(s) of SM synthesis.

The enzyme responsible for the biosynthesis of SM, SMsynthase, transfers the phosphocholine headgroup from phosphatidylcholine to ceramide. The intracellular location of SMsynthase is still a question under debate (Allan and Kallen, 1994; Koval et al., 1995; Kallen and Allan, 1995). SMsynthase activity has been attributed to the plasma membrane (Voelker and Kennedy, 1982; Marggraf et al., 1981, 1982; Marggraf and Kanfer, 1987; Futerman et al., 1990; van Helvoort et al., 1994), Golgi apparatus (Futerman et al., 1990; Lipsky and Pagano, 1983, 1985a,b; Kobayashi and Pagano, 1989; Jeckel et al., 1990; Schweizer et al., 1994) and endosomes (Allan and Kallen, 1994; Kallen et al., 1993, 1994) and, in earlier studies, to the endoplasmic reticulum (ER) (van Golde et al., 1974; Bernert and Ullman, 1981) and mitochon- dria (Sribney, 1971). In careful subfractionation studies performed on rat liver, separating plasma membrane from Golgi, the majority of the SM-synthase activity colocalized with the cis/medial-Golgi (87\%) and some with the plasma membrane (<13\%) (Futerman et al., 1990). It was concluded that SM synthesis predominantly occurs in early Golgi and that only a little SM-synthase activity is found at a post-Golgi location. More recently, Allan, Kallen and coworkers (Allan and Kallen, 1994; Kallen et al., 1994) reached an opposite conclusion from experiments in which cell surface SM was hydrolyzed by treating cells with exogenously added bacterial sphingomyelinase (SMase). The resulting ceramide was converted back to SM under conditions in which Golgi-toplasma membrane transport was inhibited. When, in addition, endocytic uptake was inhibited, resynthesis of SM was no longer observed, suggesting that efficient SM synthesis occurs in the recycling route to the plasma membrane.

The possibility that endosomes of the recycling pathway are the main site of de novo synthesis of SM, was not excluded in the subfractionation studies mentioned above, since endosomes may have localized to Golgi-containing fractions. Indeed, in many cell fractionation studies endosomes and Golgi colocalize. Only by making use of specialized techniques such as free flow electrophoresis or DAB-cytochemistry, is it possible to determine whether SM-synthase is present in the Golgi, in endosomes, or in both.

In the present paper we made use of DAB-cytochemistry (Courtoy et al., 1984; Stoorvogel et al., 1988) to separate 
endosomes from Golgi and plasma membrane. This method employs endocytosed horseradish peroxidase (HRP) which, in the presence of peroxide $\left(\mathrm{H}_{2} \mathrm{O}_{2}\right)$, converts $3,3^{\prime}$-diaminobenzidine (DAB) into a dense polymer. Enzymes present in HRPcontaining endosomes may be cross-linked to the DABpolymer and lose their activity (quenching). Furthermore, in cell fractionation experiments HRP-containing organelles equilibrate at higher densities in density gradients (shifting). We labeled the endocytic uptake and recycling pathway of the transferrin receptor using transferrin conjugated to HRP (TfHRP). The complete endocytic pathway, including lysosomes, was labeled using fluid phase endocytosed HRP (Fig. 1). Both HepG2 and BHK-21 cells were used since, using DAB-cytochemistry, the routing of Tf-HRP and HRP has been extensively studied in the former cell line (Stoorvogel et al., 1987, 1988; Rijnboutt et al., 1992; Strous et al., 1993; van Weert et al., 1995), and an endosomal localization of SM-synthase has been proposed for the latter (Allan and Kallen, 1994).

No substantial amount of SM-synthase activity was detected in the endocytic pathway of either cell line. Instead, most SMsynthase activity was found at the Golgi and less than $10 \%$ at the cell surface.

\section{MATERIALS AND METHODS}

\section{Materials}

Cell culture plastics were obtained from Costar (Cambridge, MA) and cell culture media from Gibco (Paisley, UK). Horseradish peroxidase type VI (HRP), transferrin (Tf), 3,3'-diaminobenzidine (DAB), DNase I and bovine serum albumin fraction V (BSA) were from Sigma ( $\mathrm{St}$ Louis, MO) and Percoll was obtained from Pharmacia (Uppsala, Sweden). N-6[7-nitro-2,1,3-benzoxadiazol-4-yl] amino hexanoylceramide $\left(\mathrm{C}_{6}\right.$-NBD-Cer) was from Molecular Probes (Eugene, $\mathrm{OR}$ ), $\mathrm{N}$-(lissamine rhodamine B sulfonyl)-phosphatidylethanolamine (N$\mathrm{Rh}-\mathrm{PE}$ ) from Avanti (Alabaster, AL), Desferal from CIBA-Geigy (Basel, Switzerland), and MES and silica 60 TLC plates were from Merck (Darmstadt, Germany). The NBD-analog of galactosylceramide $\left(\mathrm{C}_{6}\right.$-NBD-GalCer) was synthesized using the method described before (van Meer et al., 1987). All other chemicals and solvents were of analytical grade and obtained from Riedel-de Haën (Seelze, Germany)

${ }^{125}$ I-labeling of $\mathrm{Tf}$ and conjugation of HRP to Tf was carried out as described before (Stoorvogel et al., 1988).

\section{Cell culture}

Cells were grown and maintained as monolayer cultures on plastic dishes in a humidified atmosphere of $5 \% \mathrm{CO}_{2}$ in air. HepG2 clone A16 was cultured in MEM with 10\% FCS as described (Stoorvogel et al., 1987), and the fibroblast-like cell line BHK-21 clone C-13 (ATCC, Rockville, ML) was cultured in Glasgow MEM with $10 \%$ FCS. Cells were free of mycoplasma contamination. For experiments, semi-confluent cell monolayers on $6 \mathrm{~cm}$ dishes were used.

\section{DAB cytochemistry}

DAB-cytochemistry was principally performed according to Stoorvogel et al. (1991). Cells were washed three times and incubated for 30 minutes at $37^{\circ} \mathrm{C}$ in MEM without bicarbonate, supplemented with 20 $\mathrm{mM}$ Hepes/NaOH, pH $7.2(\mathrm{MEMH})$. Subsequently, cells were incubated in the presence of Tf-HRP $(25 \mu \mathrm{g} / \mathrm{ml})$ or HRP $(3 \mathrm{mg} / \mathrm{ml})$ in MEMH (1.5 ml) for 1 hour at $37^{\circ} \mathrm{C}$, to load the recycling route to the plasma membrane and the complete endocytic pathway, respectively. In both cases the endosomal marker ${ }^{125} \mathrm{I}-\mathrm{Tf}(2 \mu \mathrm{g} / \mathrm{ml})$ was added as a positive control to validate the DAB-induced density shift protocol. Endocytosis and recycling was stopped by washing the cells with ice-cold MEMH. Tf and Tf-HRP bound to the cell surface was removed by incubating the cells with an acidic medium followed by an alkaline medium: cells were washed sequentially at $0^{\circ} \mathrm{C}$, three times with MEMH (10 minutes in total), two times with $150 \mathrm{mM}$ $\mathrm{NaCl}, 50 \mu \mathrm{M}$ Desferal, $25 \mathrm{mM} \mathrm{MES} / \mathrm{HCl} \mathrm{pH} 5.0$ (10 minutes in total), twice with MEMH (10 minutes in total), and twice with $150 \mathrm{mM}$ $\mathrm{NaCl}, 2 \mathrm{mM} \mathrm{CaCl}, 50 \mu \mathrm{M}$ Desferal, $10 \mathrm{mM}$ glycine $\mathrm{pH} 9.0$ (30 minutes in total). The $\mathrm{pH} 9$ wash also efficiently removes extracellular HRP sticking to the plasma membrane (Geuze et al., 1988). Finally, cells were washed twice with ice-cold homogenization buffer (HB; $250 \mathrm{mM}$ sucrose, $1 \mathrm{mM}$ EDTA, $10 \mathrm{mM}$ Hepes- $\mathrm{NaOH}, \mathrm{pH}$ 7.2), scraped in $600 \mu \mathrm{lBB}$ containing $0.2 \mathrm{mM}$ PMSF, and homogenized by passage through a 23-gauge needle mounted on a syringe (10 strokes); essentially all cells were broken. Nuclei and intact cells were removed by centrifugation for 1 minute at $2,900 \mathrm{~g}$. The postnuclear supernatant (PNS) contained $70-90 \%$ of the SM-synthase activity, endosomes (internalized ${ }^{125} \mathrm{I}-\mathrm{Tf}$ ), and plasma membrane ( ${ }^{125} \mathrm{I}-\mathrm{Tf}$ or $\mathrm{N}$-Rh-PE bound in the cold, see below), and was not specifically enriched in SM-synthase activity, endosomes, or plasma membrane. The post-nuclear supernatant (PNS) of BHK-21 cells was treated with DNase I $(50 \mu \mathrm{g} / \mathrm{ml})$ for 10 minutes at $20^{\circ} \mathrm{C}$ to digest DNA released during cell homogenization. This DNA stuck to membranes and interfered with cell fractionation preventing the density-shift of HRPloaded endosomes (DNase treatment did not affect SM-synthase activity). Aliquots of PNS $(250 \mu \mathrm{l})$ were mixed with $250 \mu \mathrm{l} \mathrm{HB}$, containing both $2 \mathrm{mg} / \mathrm{ml}$ DAB and $0.04 \% \mathrm{H}_{2} \mathrm{O}_{2}$, lacking both DAB and $\mathrm{H}_{2} \mathrm{O}_{2}$, containing $\mathrm{H}_{2} \mathrm{O}_{2}$ only, or containing DAB only. After a 20 minute incubation at $20^{\circ} \mathrm{C}$, the SM-synthase activity was determined either directly (quench experiment) or after fractionation (shift experiment). For fractionation, $400 \mu \mathrm{l}$ samples were layered on top of a 7.4 $\mathrm{ml} 20 \%$ Percoll solution in $\mathrm{HB}$ and centrifuged for 25 minutes at $32,000 \mathrm{rpm}$ and $4^{\circ} \mathrm{C}$ in a Ti50 rotor (Beckman Instruments, Inc., Fullerton, CA). Twelve fractions $(650 \mu \mathrm{l})$ were collected by downward displacement. For each fraction, ${ }^{125} \mathrm{I}$-Tf was counted in a gamma-counter (LKB 1282 Wallac, Turku, Finland), and SMsynthase activity was assayed as indicated below.

\section{Determination of sphingomyelin synthesis}

SM-synthase activity was determined using the fluorescent shortchain analog $\mathrm{C}_{6}$-NBD-Cer $(10 \mu \mathrm{M})$. Aliquots of $200 \mu 1$ were mixed with $200 \mu \mathrm{l}_{6}-\mathrm{NBD}-\mathrm{Cer}$ in $\mathrm{HB}$ containing 1\% BSA (w/v), $2 \mathrm{mM}$ $\mathrm{MgCl}_{2}, 2 \mathrm{mM} \mathrm{MnCl}$, and incubated for 1 hour at $37^{\circ} \mathrm{C}$.

Lipids were extracted according to Bligh and Dyer (1959); all aqueous solutions were acidified to $10 \mathrm{mM}$ acetic acid. After collecting the organic (lower) phase, the aqueous (upper) phase was reextracted with chloroform. Organic phases were pooled, dried under $\mathrm{N}_{2}$, and applied to TLC plates using chloroform/methanol $(2: 1 \mathrm{v} / \mathrm{v})$. Lipids were separated by two-dimensional TLC, using in the first dimension chloroform:methanol:ammonia, 25\% (65:25:4 v/v), and in the second dimension chloroform:acetone:methanol:acetic acid:water (50:20:10:10:5 v/v). Fluorescent lipid spots were detected under UVlight, scraped, and quantified in a fluorimeter (Kontron, Zürich, Switzerland) as described before (van Meer et al., 1987).

\section{Markers}

Surface-bound and endocytosed ${ }^{125} \mathrm{I}$-Tf were used as markers for the plasma membrane and the endosomes in the recycling route, respectively. To label the plasma membrane, cells were incubated with $2 \mu \mathrm{g}$ ${ }^{125} \mathrm{I}-\mathrm{Tf} / \mathrm{ml}$ for 30 minutes on ice, and homogenized after extensive washing with ice-cold MEMH and HB. To label the recycling route, cells were incubated in the presence of $2 \mu \mathrm{g}{ }^{125} \mathrm{I}-\mathrm{Tf} / \mathrm{ml}$ for 1 hour at $37^{\circ} \mathrm{C}$, after which cell surface-bound ${ }^{125} \mathrm{I}$-Tf was removed (see above, under DAB-cytochemistry). ${ }^{125}$ I-radioactivity was determined using a gamma counter.

The non-exchangeable long-chain fluorescent lipid N-Rh-PE was used as a second, independent, marker for the plasma membrane (Kok 
et al., 1990); cells were incubated for 30 minutes at $0^{\circ} \mathrm{C}$ in the presence of $2 \mu \mathrm{M}$ N-Rh-PE in Hanks' balanced salt solution, supplemented with $10 \mathrm{mM}$ Hepes, $\mathrm{pH} 7.35$ (HBSS), and subsequently washed extensively with PBS and $\mathrm{HB}$ at $0^{\circ} \mathrm{C}$. After fractionation and gradient centrifugation, N-Rh-PE was extracted, separated on TLC and quantified (Burger et al., 1996).

Ceramide glucosyltransferase (CGlcT) was used as a Golgi marker (Futerman and Pagano, 1991; Jeckel et al., 1992; Strous et al., 1993). In short, gradient fractions were incubated for 1 hour at $37^{\circ} \mathrm{C}$ in $\mathrm{HB}$

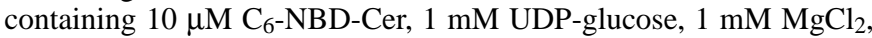
and $1 \mathrm{mM} \mathrm{MnCl}_{2}$. The resulting $\mathrm{C}_{6}$-NBD-glucosylceramide was quantified after lipid extraction and TLC (as described in Determination of sphingomyelin synthesis).

When indicated, endocytosed $\mathrm{C}_{6}$-NBD-GalCer was used as a marker for endosomes and lysosomes (Kok et al., 1991); cells were incubated for 30 minutes on ice in the presence of $5 \mu \mathrm{M} \mathrm{C}_{6}$-NBDGalCer $(0.03 \%$ BSA in MEMH). After 3 washes with PBS and 2 with MEMH at $0^{\circ} \mathrm{C}$, cells were incubated for 30 minutes at $37^{\circ} \mathrm{C}$ to allow endocytic uptake of $\mathrm{C}_{6}$-NBD-GalCer. $\mathrm{C}_{6}$-NBD-GalCer remaining at the cell surface was removed by incubating the cells in MEMH containing $2 \% \mathrm{BSA}$ for 30 minutes at $0^{\circ} \mathrm{C}$. After washing with $\mathrm{MEMH}$ and $\mathrm{HB}$, the cells were fractionated and the $\mathrm{C}_{6}-\mathrm{NBD}-\mathrm{GalCer}$ content of each gradient fraction was determined after lipid extraction and TLC analysis (see Determination of sphingomyelin synthesis).

\section{Sphingomyelin synthesis at the cell surface}

Cells were incubated at $10^{\circ} \mathrm{C}$ in $\mathrm{HBSS}$ supplemented with $1 \%(\mathrm{w} / \mathrm{v})$ BSA (HBSS+BSA), and increasing concentrations of $\mathrm{C}_{6}-\mathrm{NBD}-\mathrm{Cer}$ (van Helvoort et al., 1994). After 3 hours, the medium was refreshed and the incubation continued for 30 minutes at $10^{\circ} \mathrm{C}$. Only the $\mathrm{C}_{6-}$ NBD-SM synthesized at the cell surface is removed by the BSA present in the medium. Fluorescent lipids were extracted from the cells and the pooled media, and analyzed as described (see Determination of sphingomyelin synthesis).

\section{RESULTS}

\section{SM-synthase activity does not localize to endosomes}

To determine whether SM-synthase is localized to endosomes, DAB-cytochemistry was used. The endocytic pathway was labeled with peroxidase activity by allowing endocytic uptake of either Tf-HRP or HRP (Fig. 1). The cells were homogenized and incubated in the presence of DAB and $\mathrm{H}_{2} \mathrm{O}_{2}$. As a consequence of the HRP-activity, DAB polymerizes in the endosomes and/or lysosomes. This results in cross-linking of the endosomal protein content to the DAB-polymer (Ajioka and Kaplan, 1987; Stoorvogel et al., 1988, 1989; Rijnboutt et al., 1992), and may be accompanied by inactivation of some of the endosomal enzymes (Draye et al., 1988). In addition, the equilibrium density of endosomes is markedly increased by the presence of dense DAB-polymer. The addition of $\mathrm{H}_{2} \mathrm{O}_{2}$ alone did not affect SM synthesis in the PNS prepared from Tf-HRPor HRP-loaded HepG2, or BHK-21 cells (Fig. 2A and B, respectively). The addition of $\mathrm{DAB}$ alone also did not affect the SM-synthase activity in the PNS of HepG2 cells, but the activity increased 1.3- to 1.5-fold in the PNS of BHK-21 cells (Fig. 2B). This increase in synthase activity may be due to the insertion of the rather hydrophobic DAB into membranes, somehow stimulating SM-synthase activity. We cannot explain why this effect was observed only for BHK-21 cells and not for $\mathrm{HepG} 2$ cells. Addition of both $\mathrm{DAB}$ and $\mathrm{H}_{2} \mathrm{O}_{2}$ resulted in the deposition of DAB-polymer in HRP-containing endosomes and/or lysosomes (see below) but did not affect SM-synthase

\section{A Transferrin HRP loaded compartments}

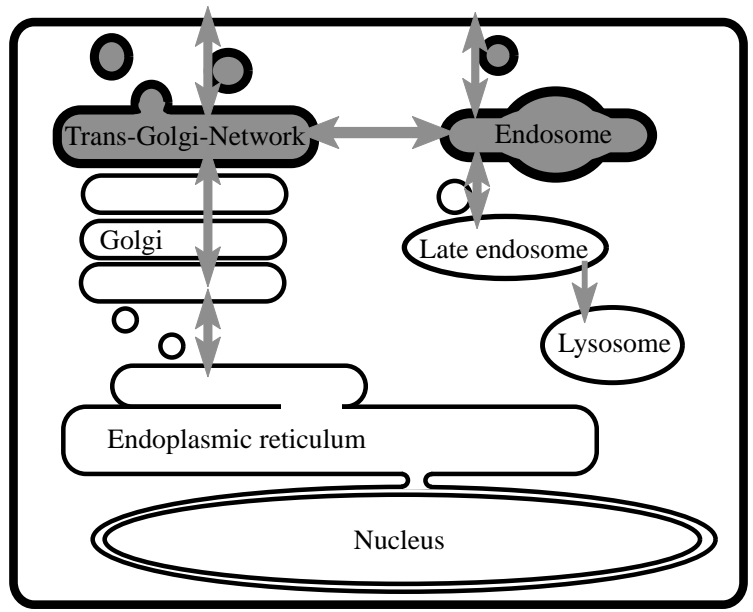

Plasma membrane

\section{B Fluid phase HRP loaded compartments}

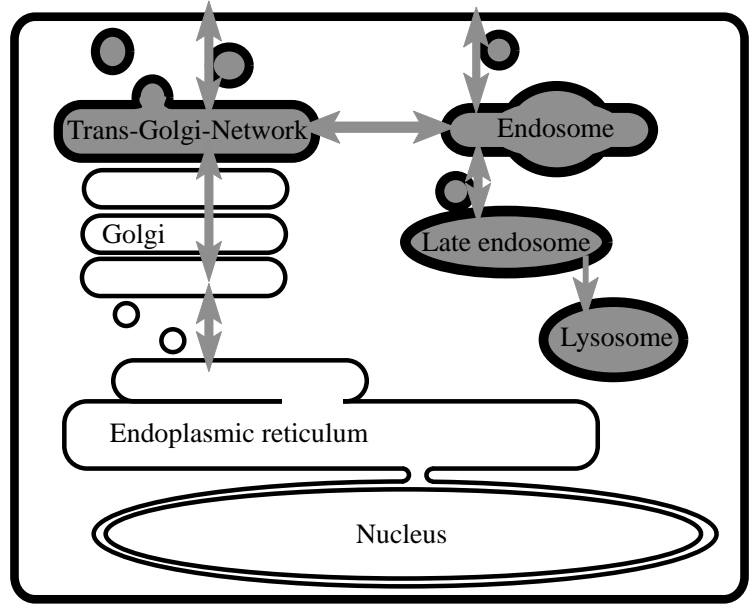

Plasma membrane

Fig. 1. Organelles loaded with HRP-activity. Illustration of the organelles loaded with peroxidase activity after internalization of either Tf-HRP (A) or of HRP (B). Loaded organelles are shaded. TfHRP behaves like unconjugated Tf (Stoorvogel et al., 1988). It binds to the transferrin receptor at the plasma membrane, is taken up by receptor-mediated endocytosis and recycles back to the plasma membrane, thus only reaching the recycling route to the plasma membrane (A). In contrast, if unconjugated 'fluid phase' HRP is used, the HRP is endocytosed reaching the plasma membrane recycling and lysosomal route (B) (Stoorvogel et al., 1988; Rijnboutt et al., 1992). The plasma membrane is not labeled because the cell surface associated HRP is removed before DAB-polymerization is initiated (see Materials and Methods).

activity as compared to addition of DAB alone. From these data we conclude that either SM-synthase is not present in the endosomes, or the activity of endosomal SM-synthase is not affected by DAB-polymerization. To exclude the latter explanation we determined whether the equilibrium density of membranes containing SM-synthase was affected by the formation of a DAB-polymer inside endosomes. 
Fig. 2. Effect of DAB-cytochemistry on SMsynthase activity. HepG2 and BHK-21 cells were loaded with Tf-HRP or HRP as described in Materials and Methods. After homogenization, samples of the PNS were incubated, in the absence of DAB and $\mathrm{H}_{2} \mathrm{O}_{2}$, in the presence of DAB only, $\mathrm{H}_{2} \mathrm{O}_{2}$ only, or both DAB and $\mathrm{H}_{2} \mathrm{O}_{2}$. SM-synthase activity was assayed as described in Materials and Methods. Data are expressed relative to $\mathrm{SM}$ synthesis in the absence of DAB and $\mathrm{H}_{2} \mathrm{O}_{2}$ (arbritary units; range with $n=2$ ).
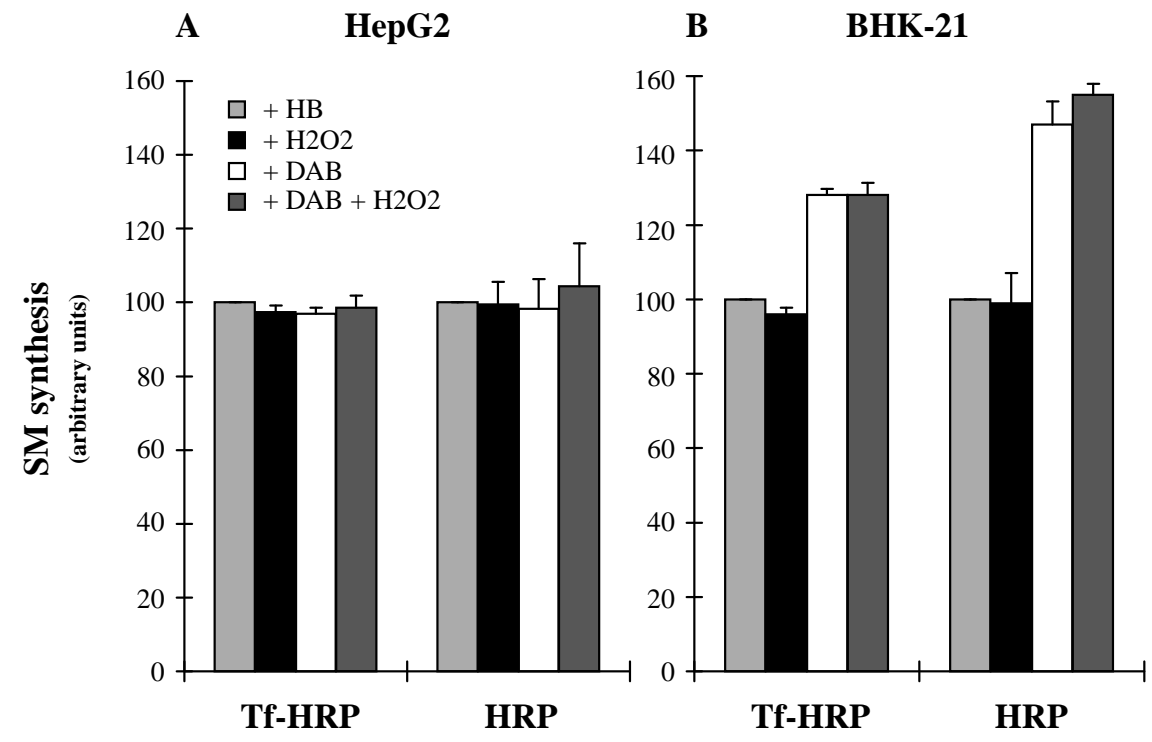

Cells were labeled with either Tf-HRP and ${ }^{125} \mathrm{I}$-Tf, or with HRP and ${ }^{125}$ I-Tf. Subsequently, the cells were homogenized and the post-nuclear supernatant (PNS) fractionated on Percoll density gradients. When the PNS was not treated with DAB and $\mathrm{H}_{2} \mathrm{O}_{2}$ prior to fractionation, the marker for early endosomes ( $\left.{ }^{125} \mathrm{I}-\mathrm{Tf}\right)$ and SM-synthase activity colocalized and peaked in fraction 7 of the Percoll density gradient (Fig. 3). When the PNS was fractionated after DAB-polymerization, 125I-Tf-labeled early endosomes shifted towards dense fractions of the gradient, both in cells loaded with Tf-HRP and in cells loaded with HRP (Fig. 3A and B, respectively). In contrast, the distribution of the SM-synthase activity did not change (Fig. 3C and D). Principally the same observations were made for BHK-21 cells. After cell fractionation on a Percoll density gradient, the marker for early endosomes $\left({ }^{125} \mathrm{I}-\right.$ Tf) and SM-synthase activity largely colocalized and peaked in fraction 6 or 7 (Fig. 4). Fractionation of the PNS after DABpolymerization revealed a clear shift of early endosomes towards the bottom of the gradient (Fig. 4A and B). However, SM-synthase activity did not shift under any of these conditions (Fig. 4C and D).

The high concentrations of Percoll in the bottom fractions
Fig. 3. Effect of DAB-cytochemistry on the density distribution of ${ }^{125} \mathrm{I}$-Tf labeled endosomes and SM-synthase containing organelles in HepG2 cells. HepG2 cells were loaded with ${ }^{125} \mathrm{I}-\mathrm{Tf}$ and either Tf-HRP (A and C) or HRP (B and D). Samples of PNS were incubated in the absence $(\square)$ or presence ( $\square$ ) of DAB and $\mathrm{H}_{2} \mathrm{O}_{2}$, and fractionated on Percoll gradients. (A and $\mathrm{B}$ ) The distribution of ${ }^{125} \mathrm{I}-\mathrm{Tf}$ loaded endosomes. (C and D) The SMsynthase activity in the same gradient. The same distribution of SM-synthase was obtained when $50 \mu \mathrm{M}$ instead of $10 \mu \mathrm{M} \mathrm{C}_{6}$-NBD-Cer was used (not shown). As a control, Percoll gradient fractions lacking membranes were mixed with a fixed volume of PNS, and the SM-synthase activity analyzed $(\triangle$ in $C)$. One out of two independent experiments, each performed in duplicate, is shown. Range of values falls within symbol size. For additional marker analysis see Fig. 5.

\section{transferrin-HRP}
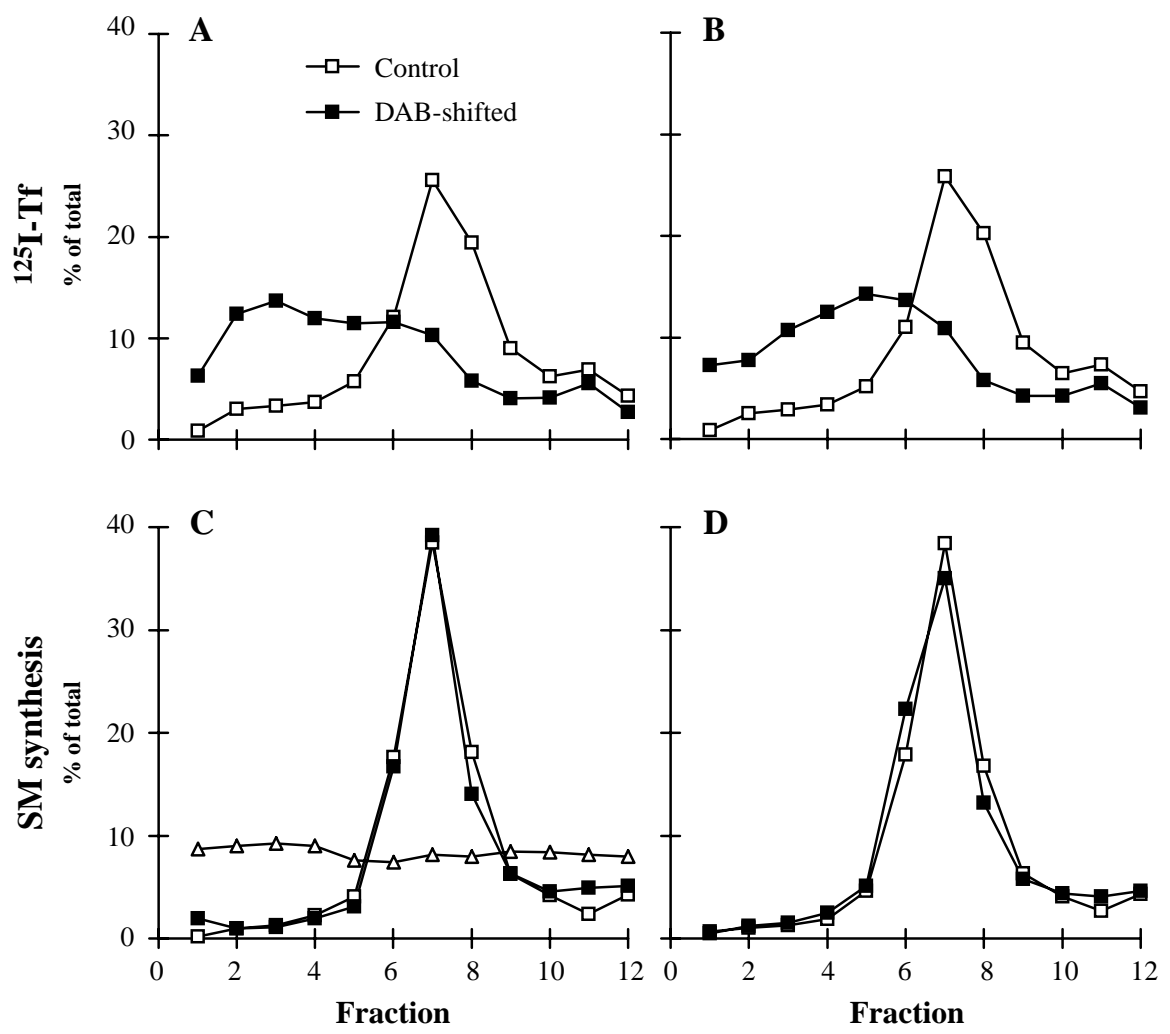


\section{transferrin-HRP}

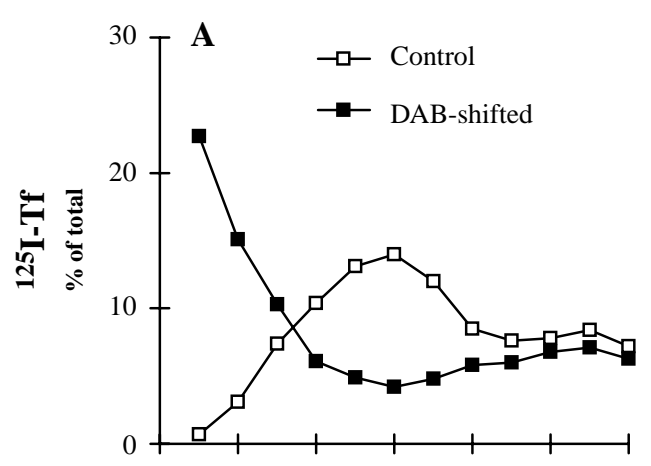

Fig. 4. Effect of DAB-cytochemistry on the density distribution of ${ }^{125}$ I-Tf-labeled endosomes and SM-synthase containing organelles in BHK-21 cells. BHK-21 cells were loaded with ${ }^{125}$ I-Tf and either Tf-HRP (A and C) or HRP (B and D). PNS samples were incubated in the presence of DAB but absence of $\mathrm{H}_{2} \mathrm{O}_{2}(\square)$, or in the presence ( $\square$ ) of both DAB and $\mathrm{H}_{2} \mathrm{O}_{2}$ and fractionated on Percoll gradients. DAB was also added to the control PNS because it slightly stimulated SM-synthase activity (see Fig. 2). (A and B) The distribution of ${ }^{125}$ I-Tf-loaded endosomes. (C and D) The distribution of SM-synthase activity in these gradients. One out of two independent experiments, each performed in duplicate, is shown. Range of values falls within symbol size. For additional marker analysis see Fig. 5.

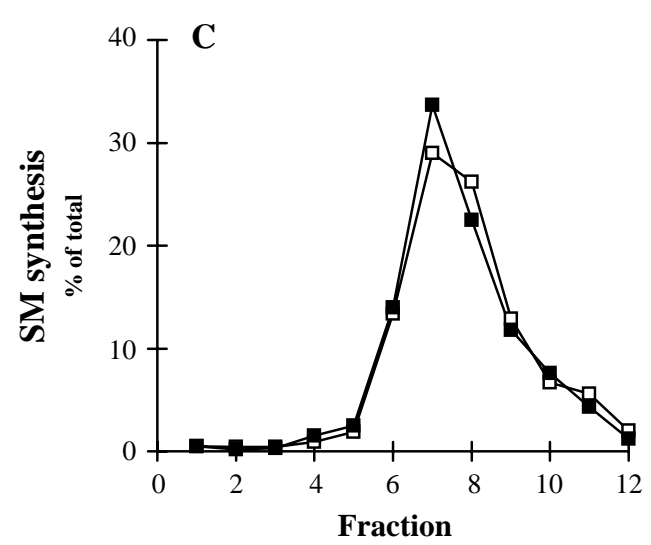

fluid phase-HRP
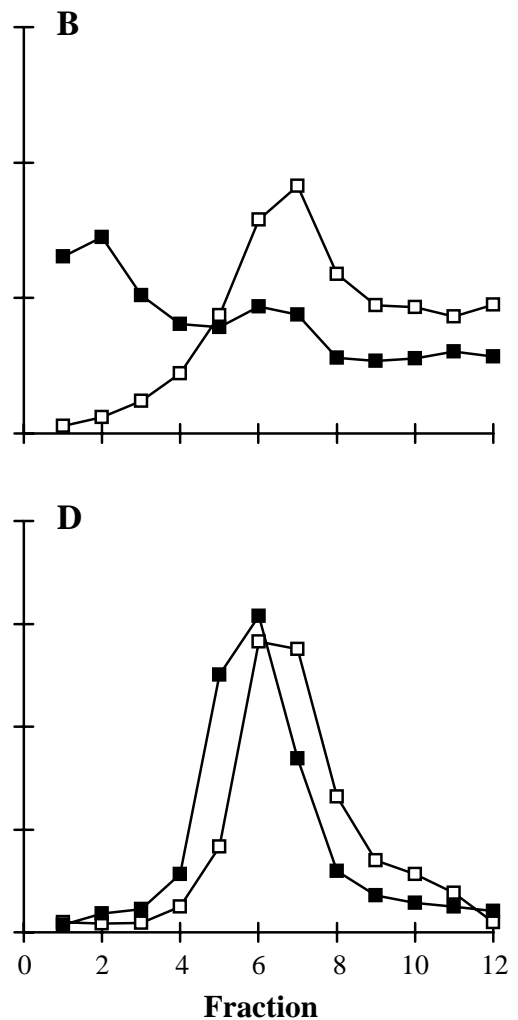

of the gradient did not mask the SM-synthase activity nor influence the efficiency of lipid extraction. This was shown in a control experiment in which fractions of a Percoll gradient that had not been loaded with PNS were mixed with PNS and assayed for SM-synthase activity (triangles in Fig. 3C). To exclude the possibility that an SM-synthase activity in endosomes is not detected because of the presence of an SMase, experiments were performed in the absence of $\mathrm{Mg}^{2+}$ and $\mathrm{Mn}^{2+}$, but in the presence of $1 \mathrm{mM}$ EDTA to inhibit the neutral SMase (Futerman et al., 1990): an effect on SMsynthase activity was not found, not on the gradient profile nor on the total amount of synthesis (data not shown). The results are also not affected by the activity of the acidic SMase present in endosomes because this enzyme is inactive at the assay conditions used (at pH 7.2 and in the absence of ATP the endosome is $\mathrm{pH}$ neutral).

We conclude that in HepG2 and BHK-21 cells SM-synthase is not located in significant amounts in organelles reached by Tf-HRP or HRP, i.e. the early or late endosomes and lysosomes.

\section{SM synthesis at the cell surface}

Our data clearly indicate that SM-synthase is absent from the endocytic route, but do not exclude other post-Golgi locations of SM-synthase. Futerman et al. (1990) found that part of the SM-synthase activity cofractionated with the plasma membrane. Assuming that the same enzyme is present in the Golgi and plasma membrane, the lumenal orientation of SMsynthase in the Golgi (Futerman et al., 1990; Futerman and Pagano, 1991; Jeckel et al., 1992) predicts that the SMsynthase in the plasma membrane has its active center at the cell surface. Indeed, we concluded from experiments performed at low temperature that part of the SM-synthase activity was present at the outer leaflet of the plasma membrane (van Helvoort et al., 1994). Because the plasma membrane was not separated from the Golgi in Percoll density gradients (Fig. 5), we used the low temperature assay to determine the relative SM-synthase activity at the cell surface. At $10^{\circ} \mathrm{C}$, short-chain $\mathrm{C}_{6}$-NBD-Cer permeates through membranes and reaches all possible locations of SM-synthase activity within the cell and at the cell surface. However, at this temperature intracellularly synthesized $\mathrm{C}_{6}$-NBD-SM does not reach the cell surface because vesicular transport is inhibited. Therefore, only the $\mathrm{C}_{6}$ NBD-SM made at the cell surface is removed from the cells by BSA in the incubation medium. Since the amount of $\mathrm{C}_{6}$ NBD-SM synthesized can be expected to be dependent on the local concentration of $\mathrm{C}_{6}-\mathrm{NBD}-\mathrm{Cer}$, and the availability of $\mathrm{C}_{6}$ NBD-Cer may be higher at the plasma membrane compared to the Golgi, we determined the $\mathrm{C}_{6}-\mathrm{NBD}-\mathrm{SM}$ synthesis as a function of the $\mathrm{C}_{6}$-NBD-Cer concentration. Fig. 6 (insert) shows the relative amount of $\mathrm{C}_{6}-\mathrm{NBD}_{-} \mathrm{SM}$ synthesized at the cell surface as a function of the $\mathrm{C}_{6}$-NBD-Cer concentration used. Under conditions where both the intracellular and the cell surface SM-synthase activities were saturated we detected about $4 \%$ of the SM-synthase activity at the cell surface of HepG 2 cells, while this was $8 \%$ in BHK-21 cells (Fig. 6). We conclude that a small proportion of SM-synthase activity is located at the cell surface.

\section{DISCUSSION}

In subfractionation studies, SM-synthase was found to colo- 
Fig. 5. Localization of the plasma membrane and of SM-synthase activity on a Percoll gradient. HepG2 (A) and BHK-21 cells (B) were fractionated on Percoll gradients, and the distribution of SMsynthase ( $\square$ ) and markers for the early Golgi ( $\square$ ), the plasma membrane $(O)$ and the endosomes/lysosomes (O) were determined. The ceramide glucosyltransferase (CGlcT) was used as a Golgi marker; in HepG2 cells, this enzyme has been shown to be located in the early Golgi (Futerman and Pagano, 1991; Jeckel et al., 1992; Strous et al., 1993; Burger et al., 1996). To label the plasma membrane, cells were incubated at $0^{\circ} \mathrm{C}$ with the non-exchangeable fluorescent lipid N-Rh-PE. The same result was obtained analyzing the gradient distribution of ${ }^{125} \mathrm{I}-\mathrm{Tf}$ bound to the cell surface (not shown). The distribution of endosomes and lysosomes in HepG2 cells was determined using $\mathrm{C}_{6}-\mathrm{NBD}-\mathrm{GalCer}$ inserted into the plasma membrane and allowing internalization; $\mathrm{C}_{6}$-NBDGalCer follows the lysosomal route (Kok et al., 1991). Endosomes were reached by both $\mathrm{C}_{6}$-NBDGalCer and ${ }^{125} \mathrm{I}-\mathrm{Tf}$ (Fig 3 and 4), but lysosomes only by $\mathrm{C}_{6}-\mathrm{NBD}-\mathrm{GalCer}$, and peaked in fraction 2 .
A HepG2

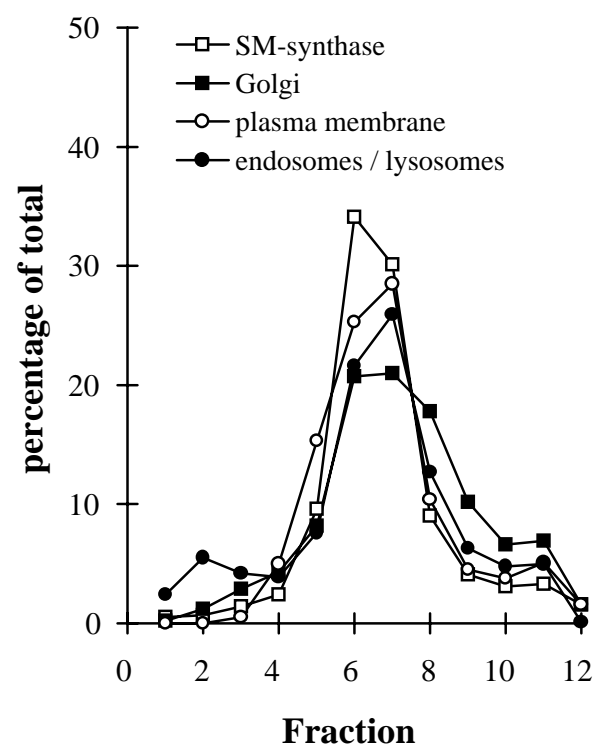

Fraction
B BHK-21

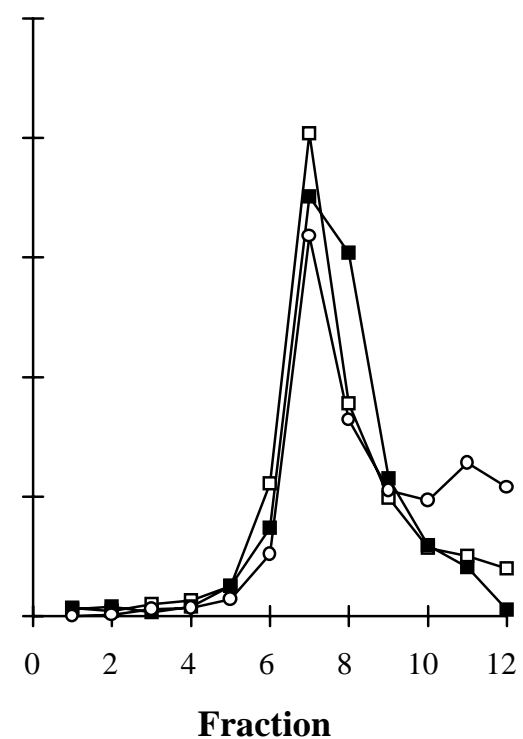

calize with early Golgi markers (Futerman et al., 1990; Jeckel et al., 1990). However, these subfractionation experiments did not exclude an endosomal localization of SM-synthase; endosomal markers were not analyzed and endosomes and Golgi often colocalize on density gradients. Recently, Allan, Kallen and coworkers indeed suggested that the major SM pool, plasma membrane SM, is synthesized in the plasma membrane recycling route (Allan and Kallen, 1994; Kallen et al., 1993). We used DAB-cytochemistry to separate endosomes from Golgi, and determine whether SM-synthase is present in endosomes, in the Golgi, or in both.

HepG2 and BHK-21 cells were loaded with Tf-HRP or HRP and DAB-polymerization was induced after cell homogenization. We show that the SM-synthase is not inactivated (Fig. 2) or shifted on density gradients (Figs 3 and 4) upon DAB-polymerization in endosomes or lysosomes. Since Tf-HRP efficiently labeled the early endosomes of the recycling route to the plasma membrane, and HRP reached the complete endocytic route including late endosomes and lysosomes (Stoorvogel et al., 1988; Rijnboutt et al., 1992; Strous et al., 1993), our data indicate that little, if any, SM-synthase is present in the endocytic pathway. An additional argument against the presence of SM-synthase in lysosomes is the almost complete absence of SM-synthase activity at the high density fractions of the Percoll gradient where lysosomes fractionate (Fig. 5; see also van Weert et al., 1995 and Stoorvogel et al., 1991). Finally, in HepG2 cells endocytosed Tf-HRP and HRP are also transported to the trans-Golgi network (TGN) (Stoorvogel et al., 1988; Rijnboutt et al., 1992; Strous et al., 1993), suggesting that at least in this cell line SM-synthase is not present in the TGN. The absence of SM-synthase activity from the TGN of HepG2 cells is consistent with other experiments: in brefeldin A-sensitive cell lines, brefeldin A induces transfer of endosomal markers to the TGN (Lippincott-Schwartz et al.,
Fig. 6. SM-synthase activity at the cell surface. HepG2 and BHK-21 cells were incubated for 3 hours at $10^{\circ} \mathrm{C}$ with varying concentrations of $\mathrm{C}_{6-}$

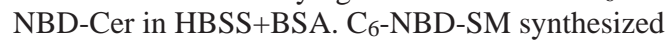
at the plasma membrane was extracted by the BSA in the medium and is plotted against the concentration of $\mathrm{C}_{6}-\mathrm{NBD}-\mathrm{Cer}$ applied. The insert shows the total amount of $\mathrm{C}_{6}$-NBD-SM synthesis as a function of the concentration of $\mathrm{C}_{6}-\mathrm{NBD}$-Cer used. (mean \pm s.d., two independent experiments, each in duplicate). Under saturating conditions less than $1 \%$ of the $\mathrm{C}_{6}-\mathrm{NBD}-\mathrm{GlcC}$ er reached the cell surface.

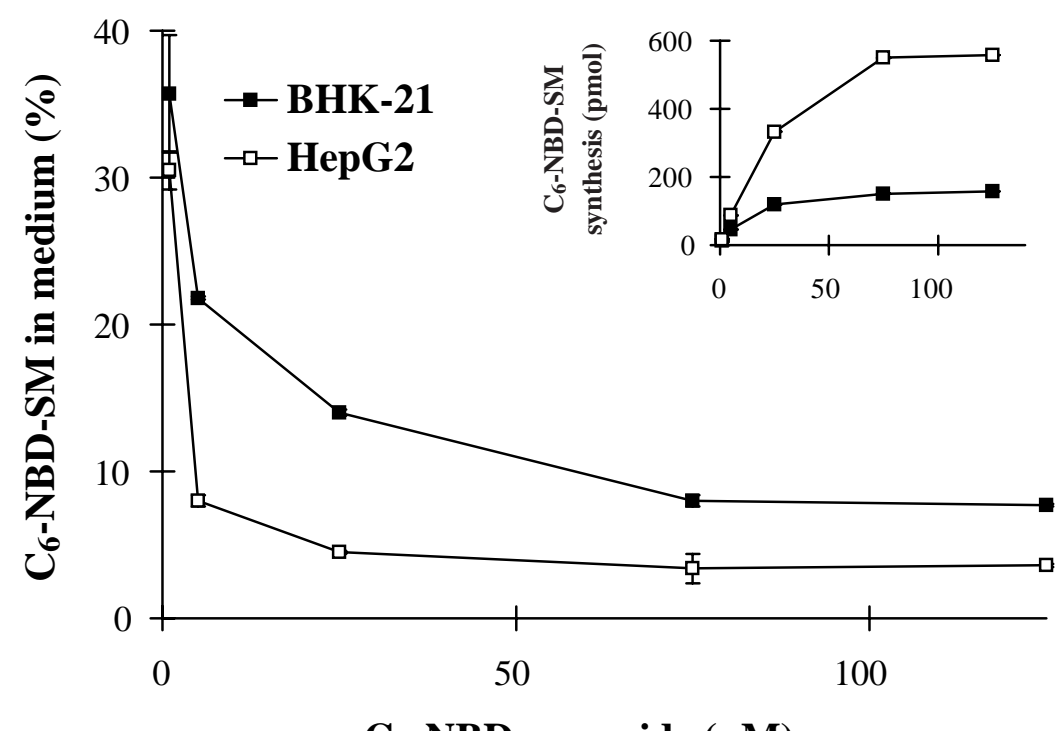

C6-NBD-ceramide $(\mu \mathrm{M})$ 
1991). If HepG2 cells loaded with HRP were incubated with brefeldin A in the continuous presence of HRP and a DABpolymer was allowed to form, SM-synthase activity was not quenched and the enzyme profile on a Percoll gradient unchanged (data not shown).

In experiments performed at low temperature under conditions in which both the intracellular sites of SM-synthase and SM-synthase at the cell surface were saturated with $\mathrm{C}_{6}-\mathrm{NBD}$ Cer, less than $10 \%$ of SM-synthase activity was found at the cell surface (Fig. 6). The reason that SM-synthase was detected at the cell surface and not in the endosomes may be explained by the absence of an internalization signal. Of plasma membrane proteins lacking an internalization signal, only about $10 \%$ is present in endosomes (Courtoy, 1991).

Since over $90 \%$ of SM-synthase activity is located inside the cell and the enzyme is absent from the endocytic route and TGN, we conclude, in agreement with the subfractionation data available from the literature (Lipsky and Pagano, 1985a; Futerman et al., 1990; Jeckel et al., 1990; Schweizer et al., 1994), that the majority of the SM-synthase activity is located in the Golgi. A small proportion of SM-synthase activity, 4\% in HepG2 and $8 \%$ in BHK-21 cells, is localized at the exoplasmic leaflet of the plasma membrane. Currently there is no evidence available indicating that de novo synthesized ceramide, the substrate of SM-synthase, is present at the plasma membrane. Ceramide is synthesized in the ER, and therefore expected to reach the Golgi more readily than the plasma membrane. Thus the relative contribution of cell surface SM-synthase in the biosynthesis of SM may be much less than $4-8 \%$. However, surface SM-synthase may play a role in signal transduction at the plasma membrane. Cell activation often results in the stimulation of a plasma membrane SMase generating high concentrations of ceramide presumably in the cytosolic leaflet of the plasma membrane (Linardic and Hannun, 1994; Andrieu et al., 1996). This ceramide can easily translocate across the plasma membrane and reach the SMsynthase at the exoplasmic leaflet. Thus, SM-synthase may have an important function in removing ceramide from the plasma membrane and releasing diacylglycerol. Interestingly, SM-synthase may also be capable of catalyzing the reverse reaction, the formation of phosphatidylcholine from diacylglycerol and SM, and may therefore regulate the concentration in the plasma membrane of the two important second messengers, ceramide and diacylglycerol (van Helvoort et al., 1994).

The conclusion that the major site of SM-synthase is located in endosomes in the recycling route (Allan and Kallen, 1994; Kallen et al., 1993) was based on experiments in which cells were treated with bacterial SMase yielding high concentrations of ceramide in the exoplasmic leaflet of the plasma membrane. This ceramide was found to be converted back to SM even in cells that were pretreated with brefeldin A (Allan and Kallen, 1994; Kallen et al., 1993), a drug that inhibits vesicular traffic between the Golgi and plasma membrane. Because surface SM-synthase is not inhibited by brefeldin A (data not shown), we consider it likely that resynthesis of SM in brefeldin Atreated cells is catalyzed by the SM-synthase at the cell surface, and not by a SM-synthase in the endocytic route. However, we have currently no explanation for why resynthesis of SM was inhibited in mitotic and energy-depleted cells, in which SMsynthase activity at the cell surface is expected to be normal.

Together, our data are consistent with the literature
(Futerman et al., 1990; Jeckel et al., 1990), and strongly indicate that SM-synthase is present in the Golgi and at the plasma membrane, and is virtually absent from the endocytic route and the TGN. Unfortunately, attempts to purify SMsynthase or clone its encoding DNA have not been successful so far. This purification is an important objective for future research, since it will allow antibodies to be generated against the protein. These antibodies will be useful to confirm the intracellular distribution of SM-synthase by immuno-electron microscopy. SM-synthase-encoding cDNA could be used to screen for homologous proteins and answer the question of whether the SM-synthase in the Golgi and plasma membrane are identical or whether different isoenzymes are present at different locations. In the end this will allow us to better understand how the SM content of the various intracellular membranes and the plasma membrane is regulated.

We are very grateful to Anton van Weert for critical advice and technical support. The present work was supported by a fellowship from the Netherlands Foundation for Chemical Research with financial aid from the Netherlands Organization for Scientific Research (NWO) to A. van Helvoort, and by the European Community contract BIO2-CT93-0348 to G. van Meer and K.N.J. Burger.

\section{REFERENCES}

Ajioka, R. S. and Kaplan, J. (1987). Characterization of endocytic compartments using the horseradish peroxidase-diaminobenzidine density shift technique. J. Cell Biol. 104, 77-85.

Allan, D. and Kallen, K.-J. (1994). Is plasma membrane lipid composition defined in the exocytic or the endocytic pathway? Trends Cell Biol. 4, 350353.

Andrieu, N., Salvayre, R. and Levade, T. (1996). Comparative study of the metabolic pools of sphingomyelin and phosphatidylcholine sensitive to tumor necrosis factor. Eur. J. Biochem. 236, 738-745.

Bernert, J. T. and Ullman, M. D. (1981). Biosynthesis of sphingomyelin from erythro-ceramides and phosphatidylcholine by a microsomal cholinephosphotransferase. Biochim. Biophys. Acta 666, 99-109.

Bligh, E. G. and Dyer, W. J. (1959). A rapid method of total lipid extraction and purification. Can. J. Biochem. Physiol. 37, 911-917.

Burger, K. N. J., van der Bijl, P. and van Meer, G. (1996). Topology of sphingolipid galactosyltransferases in ER and Golgi: transbilayer movement of monohexosyl sphingolipids is required for higher glycosphingolipid biosynthesis. J. Cell Biol. 133, 15-28.

Courtoy, P. J., Quintart, J. and Baudhuin, P. (1984). Shift of equilibrium density induced by 3,3'-diaminobenzidine cytochemistry: a new procedure for the analysis and purification of peroxidase-containing organelles. J. Cell Biol. 98, 870-876.

Courtoy, P. J. (1991). Dissection of endosomes. In Intracellular Trafficking of Proteins (ed. J. C. Steer and J. Harford), pp. 103-156. Cambridge University Press, New York.

Draye, J.-P., Courtoy, P. J., Quintart, J. and Baudhuin, P. (1988). A quantitative model of traffic between plasma membrane and secondary lysosomes: evaluation of inflow, lateral diffusion, and degradation. J. Cell Biol. 107, 2109-2115.

Futerman, A. H., Stieger, B., Hubbard, A. L. and Pagano, R. E. (1990) Sphingomyelin synthesis in rat liver occurs predominantly at the cis and medial cisternae of the Golgi apparatus. J. Biol. Chem. 265, 8650-8657.

Futerman, A. H. and Pagano, R. E. (1991). Determination of the intracellular sites and topology of glucosylceramide synthesis in rat liver. Biochem. J. 280, 295-302.

Geuze, H. J., Stoorvogel, W., Strous, G. J., Slot, J. W., Bleekemolen, J. E. and Mellman, I. (1988). Sorting of mannose 6-phosphate receptors and lysosomal membrane proteins in endocytic vesicles. J. Cell Biol. 107, 24912501 .

Jeckel, D., Karrenbauer, A., Birk, R., Schmidt, R. R. and Wieland, F. (1990). Sphingomyelin is synthesized in the cis Golgi. FEBS Lett. 261, 155 157. 
Jeckel, D., Karrenbauer, A., Burger, K. N. J., van Meer, G. and Wieland, F. (1992). Glucosylceramide is synthesized at the cytosolic surface of various Golgi subfractions. J. Cell Biol. 117, 259-267.

Kallen, K.-J., Quinn, P. and Allan, D. (1993). Monensin inhibits synthesis of plasma membrane sphingomyelin by blocking transport of ceramide through the Golgi: evidence for two sites of sphingomyelin synthesis in BHK cells. Biochim. Biophys. Acta 1166, 305-308.

Kallen, K.-J., Allan, D., Whatmore, J. and Quinn, P. (1994). Synthesis of surface sphingomyelin in the plasma membrane recycling pathway of BHK cells. Biochim. Biophys. Acta 1191, 52-58.

Kallen, K.-J. and Allan, D. (1995). Sphingomyelin synthesis in endosomal compartments? Reply. Trends Cell Biol. 5, 149.

Kobayashi, T. and Pagano, R. E. (1989). Lipid transport during mitosis. Alternative pathways for delivery of newly synthesized lipids to the cell surface. J. Biol. Chem. 264, 5966-5973.

Kok, J. W., ter Beest, M., Scherphof, G. and Hoekstra, D. (1990). A nonexchangeable fluorescent phospholipid analog as a membrane traffic marker of the endocytic pathway. Eur. J. Cell Biol. 53, 173-184.

Kok, J. W., Babia, T. and Hoekstra, D. (1991). Sorting of sphingolipids in the endocytic pathway of HT29 cells. J. Cell Biol. 114, 231-239.

Koval, M., Futerman, A. H. and Pagano R. E. (1995). Sphingomyelin synthesis in endosomal compartments? Trends Cell Biol. 5, 148-149.

Linardic, C. M. and Hannun, Y. A. (1994). Identification of a distinct pool of sphingomyelin involved in the sphingomyelin cycle. J. Biol. Chem. 269, 23530-23537.

Lippincott-Schwartz, J., Yuan, L., Tipper, C., Amherdt, M., Orci, L. and Klausner, R. D. (1991). Brefeldin A's effects on endosomes, lysosomes, and the TGN suggest a general mechanism for regulating organelle structure and membrane traffic. Cell 67, 601-616.

Lipsky, N. G. and Pagano, R. E. (1983). Sphingolipid metabolism in cultured fibroblasts: microscopic and biochemical studies employing a fluorescent ceramide analogue. Proc. Nat. Acad. Sci. USA 80, 2608-2612.

Lipsky, N. G. and Pagano, R. E. (1985a). Intracellular translocation of fluorescent sphingolipids in cultured fibroblasts: Endogenously synthesized sphingomyelin and glucocerebroside analogues pass through the Golgi apparatus en route to the plasma membrane. J. Cell Biol. 100, 27-34.

Lipsky, N. G. and Pagano, R. E. (1985b). A vital stain for the Golgi apparatus. Science 228, 745-747.

Marggraf, W.-D., Anderer, F. A. and Kanfer, J. N. (1981). The formation of sphingomyelin from phosphatidylcholine in plasma membrane preparations from mouse fibroblasts. Biochim. Biophys. Acta 664, 61-73.

Marggraf, W.-D., Zertani, R., Anderer, F. A. and Kanfer, J. N. (1982). The role of endogenous phosphatidylcholine and ceramide in the biosynthesis of sphingomyelin in mouse fibroblasts. Biochim. Biophys. Acta 710, 314-323.

Marggraf, W.-D. and Kanfer, J. N. (1987). Kinetic and topographical studies of the phosphatidylcholine:ceramide choline phosphotransferase in plasma membrane particles from mouse ascites cells. Biochim. Biophys. Acta 897, $57-68$.
Rijnboutt, S., Stoorvogel W., Geuze, H. J. and Strous, G. J. (1992) Identification of subcellular compartments involved in biosynthetic processing of cathepsin D. J. Biol. Chem. 267, 15665-15672.

Schweizer, A., Clausen, H., van Meer, G. and Hauri, H. P. (1994). Localization of $O$-glycan initiation, sphingomyelin synthesis, and glucosylceramide synthesis in Vero cells with respect to the endoplasmic reticulum-Golgi intermediate compartment. J. Biol. Chem. 269, 4035-4041.

Sribney, M. (1971). Stimulation of sphingomyelin synthetase by sulfhydryl reagents. Can. J. Biochem. 49, 306-310.

Stoorvogel, W., Geuze, H. J. and Strous, G. J. (1987). Sorting of endocytosed transferrin and asialoglycoprotein occurs immediately after internalization in HepG2 cells. J. Cell Biol. 104, 1261-1268.

Stoorvogel, W., Geuze, H. J., Griffith, J. M. and Strous, G. J. (1988). The pathways of endocytosed transferrin and secretory protein are connected in the trans-Golgi reticulum. J. Cell Biol. 106, 1821-1829.

Stoorvogel, W., Geuze, H. J., Griffith, J. M., Schwartz, A. L. and Strous, G. J. (1989). Relations between the intracellular pathways of the receptors for transferrin, asialoglycoprotein, and mannose 6-phosphate in human hepatoma cells. J. Cell Biol. 108, 2137-2148.

Stoorvogel, W., Strous, G. J., Geuze, H. J., Oorschot, V. and Schwartz, A. L. (1991). Late endosomes derive from early endosomes by maturation. Cell 65, 417-427.

Strous, G. J., van Kerkhof, P., van Meer, G., Rijnboutt, S. and Stoorvogel, W. (1993). Differential effects of brefeldin A on transport of secretory and lysosomal proteins. J. Biol. Chem. 268, 2341-2347.

van den Hill, A., van Heusden, G. P. H. and Wirtz, K. W. A. (1985). The synthesis of sphingomyelin in the Morris hepatomas 7777 and 5123D is restricted to the plasma membrane. Biochim. Biophys. Acta 833, 354-357.

van Golde, L. M. G., Raben, J., Batenburg, J. J, Fleischer, B., Zambrano, F. and Fleischer, S. (1974). Biosynthesis of lipids in Golgi complex and other subcellular fractions from rat liver. Biochim. Biophys. Acta 360, 179-192.

van Helvoort, A., van 't Hof, W., Ritsema, T., Sandra, A. and van Meer, G. (1994). Conversion of diacylglycerol to phosphatidylcholine on the basolateral surface of epithelial (Madin-Darby canine kidney) cells. Evidence for the reverse action of a sphingomyelin synthase. J. Biol. Chem. 269, 1763-1769.

van Meer, G., Stelzer, E. H. K., Wijnaendts-van-Resandt, R. W. and Simons, K. (1987). Sorting of sphingolipids in epithelial (Madin-Darby canine kidney) cells. J. Cell Biol. 105, 1623-1635.

van Weert, A. W. M., Dunn, K. W., Geuze, H. J., Maxfield, F. R. and Stoorvogel, W. (1995). Transport from late endosomes to lysosomes, but not sorting of integral membrane proteins in endosomes, depends on the vacuolar proton pump. J. Cell Biol. 130, 821-834.

Voelker, D. R. and Kennedy, E. P. (1982). Cellular and enzymic synthesis of sphingomyelin. Biochemistry 21, 2753-2759.

(Received 16 October 1996 - Accepted 14 January 1997) 